\title{
GENERAL PACKET RADIO SERVICE PHYSICAL-LAYER PERFORMANCE SIMULATION
}

\author{
Ailton Akira Shinoda ${ }^{1}$ \\ Received may 23, 2003, accepted december 31, 2003
}

\begin{abstract}
In this paper, we present some results on GPRS physical-layer performance based on a GPRS simulator. The simulator strictly abides by the GSM standards implementing the detailed transmission chain. We analyze the results of a comprehensive simulation of the GPRS traffic channels. We evaluate the GPRS performance under various environment and service rates. The reference channel model used for the simulations is entirely based on the Ricean channel model and provides a reasonable scenario for radio propagation. The results shown here provide an insight into how to assess the actual GPRS data channels performance under various service rates.
\end{abstract}

Keywords: GPRS, GSM, simulation, wireless

\section{INTRODUCTION}

Nowadays the current growth rates of data traffic in fixed communication environments are extrapolated for wireless environment. It is expected that there will be a significant demand for wireless data services in the near future. It seems that the success of wireless data at the moment is just prohibited because certain requirements for data rates and cost are not fulfilled. But it is not visionary to predict that this will change with enhanced data services e.g. for GSM with HSCSD (High Speed Circuit Switched Data), GPRS (General Packet Radio Service), EDGE (Enhanced Data Rates for GSM Evolution), and UMTS (Universal Mobile Telecommunication System).

GSM already supports data users with circuit switched services covering data rates from 1.2 to $64 \mathrm{kbits} / \mathrm{s}$ (with HSCSD). GPRS takes off to offer improved services for data users in mobile networks. GPRS is the packetoriented extension of GSM. It allows data transmission up to rates of more than $100 \mathrm{kbps}$. Since GPRS operates as a packet-oriented extension, many users can share the scarce radio resources. In this way a very flexible access is possible while idle users can still be online always and anywhere. A detailed description of GPRS is given in [1-4].

The access scheme for GPRS is frequency and time division system (FDD/TDD), each physical channel being characterized by a carrier fequency and a time slot number with channel widths of $200 \mathrm{KHz}$. Each carrier is divided into frames of 8 time slots, with a frame duration of about $4.6 \mathrm{~ms}$. In this paper, we describe the implementation of a GPRS simulator operating in the FDD/TDD mode. Its is built as add-on modules for Simulink ${ }^{\mathrm{TM}}$ so as to allow for an easy integration of the GPRS models and the algorithms and techniques developed within Matlab ${ }^{\mathrm{TM}}$. The simulator implements in detail and rigorously all the physicallayer aspects of the GPRS, as specified in GSM recommendations Series (e.g., [5-9] ).

In section GPRS System we introduce the GPRS link transmission chain. In section Channel we discuss the Ricean channel model. In section Simulation Setup we discuss the simulation configurations concerning the simulation setups for each type of environment operation. In section Simulation Results we illustrate some results for the proposed simulation setups and analyze the GPRS performance. In section Conclusion we draw the final comments and conclusions about the actual status of this work.

\section{GPRS SYSTEM}

This section describes the GPRS as implemented in the simulator. The simulation of the GPRS is divided in two main components: 1) the implementation of the GSM standards for the transmitter and 2) the implementation of a suitable receiver matched to the transmitter specifications. The other relevant aspect within the simulation is the channel model to be used. In this paper, the Ricean channel model is included.

\footnotetext{
${ }^{1}$ The author is with the Department of Electrical Engineering, Londrina State University, Londrina, Paraná-Brazil (e-mail:shinoda@uel.br)
} 
Fig.1 shows the framework of he implemented GPRS simulator based on the GSM standards.

In GPRS radio interface, for each $20 \mathrm{~ms}$ the Coding Scheme (CS) gives the information rate from Media Access Control (MAC). The details of the codes are shown in Table I.

Each CS uses, in this order, the following sequence of operations:

- The information bits are coded with systematic block code, building words of information + parity bits.

- These information + parity bits are encoded with a convolutional code, building the coded bits.

- Reordering and interleaving the coded bits, and adding a stealing flag, gives the interleaved bits.

All those operations are made block by block, the size of which depends on the CS. However, most of the 456 coded bits, are interleaved and mapped onto bursts in a very similar way for all of them. Fig. 2 and Fig. 3 are diagrams showing the general structure of the CS scheme.

The first step in the block codification process is the pre-coding USF (Uplink State Flag) [7]. The first 3 bits from CS are coded in the 3 (CS1), 6 (CS2 or CS3) or 12 (CS4) bits. The next step performs the Block Check
Sequence (40 bits for CS1 and 16 bits for CS2-CS4) and addition of 4 tail bits (CS1-CS3). In the following is evaluated the convolutional code (CS1-CS3), which is a powerful channel coding scheme for wireless mobile communications. The convolutional encoder consists of shift registers and modulo-2 adders connected to some of the shift registers. The performance of convolutional code depends on two parameters - coding rate (r) and constraint length (M). The input sequence is fed to the $\mathrm{M}$-stage shift registers, and output data is calculated using the contents of M-stage shift registers. The generator polynomials determine the encoding process [8]. After this, the puncturing block is applied (CS2 and CS3) taking into account the match rate. Because convolutional codes perform poorly on bursts errors, interleaving is used in the transmitter to randomize the errors so that the convolutional codes can correct them. The purpose of the interleaving algorithm is to avoid loss of the consecutive information bits. The result of the interleaving is a distribution of the reordered 114 bits of a given block, in a diagonal way over consecutive blocks [8]. The following block, mapping on a burst, adds bits in the block interleaved with values pre-defined to identify the codification (CS1-CS4) scheme employed. The procedure applied in the mapping was based on [8]. The burst formatting block includes 26 training sequence bits, which are defined as modulating bits with states as given in [1] according to the training sequence code (TSC). Finally, in the transmission subsystem, the GSMK block performs the modulation, taking into account [9].

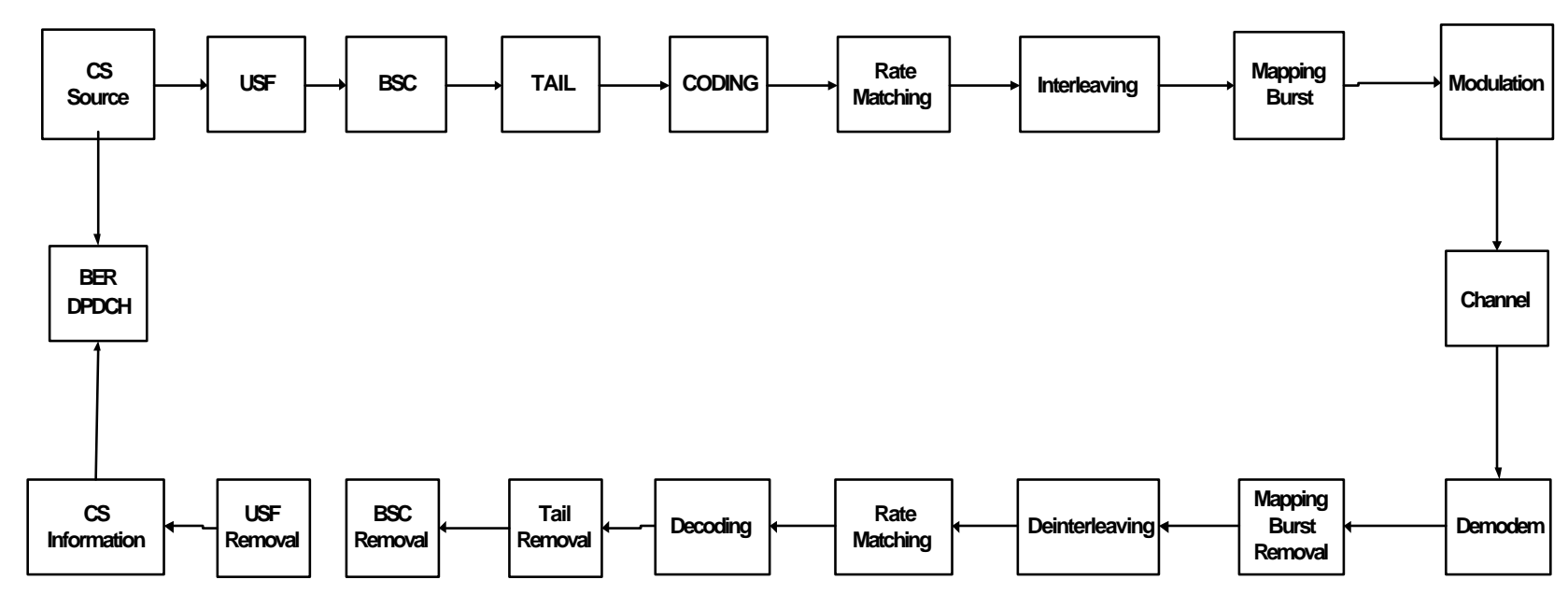

Fig. 1.- GPRS Simulator Structure 
Table 1.- GPRS Coding Scheme

\begin{tabular}{|c|c|c|c|c|c|c|c|c|}
\hline Scheme & $\begin{array}{c}\text { Code } \\
\text { rate }\end{array}$ & Payload & BCS & $\begin{array}{c}\text { Pre-coded } \\
\text { USF }\end{array}$ & Tail bits & $\begin{array}{c}\text { Coded } \\
\text { bits }\end{array}$ & $\begin{array}{c}\text { Puncturing } \\
\text { bits }\end{array}$ & $\begin{array}{c}\text { Data rate } \\
(\mathrm{kbps})\end{array}$ \\
\hline $\mathrm{CS} 1$ & $1 / 2$ & 181 & 40 & 3 & 4 & 456 & 0 & 9.05 \\
\hline $\mathrm{CS} 2$ & $\approx 2 / 3$ & 268 & 16 & 6 & 4 & 588 & 132 & 13.4 \\
\hline $\mathrm{CS} 3$ & $\approx 3 / 4$ & 312 & 16 & 6 & 4 & 676 & 220 & 15.6 \\
\hline $\mathrm{CS} 4$ & 1 & 428 & 16 & 12 & 0 & 456 & 0 & 21.4 \\
\hline
\end{tabular}

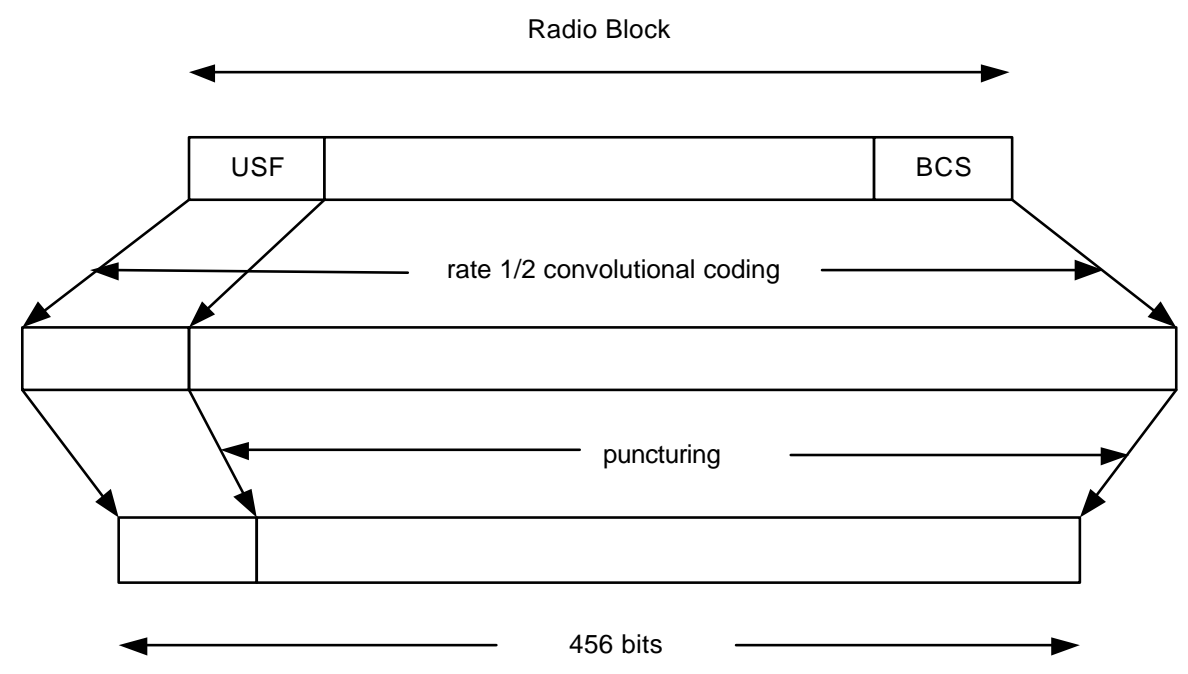

Fig. 2.- Structure of the CS scheme (CS1-CS3)

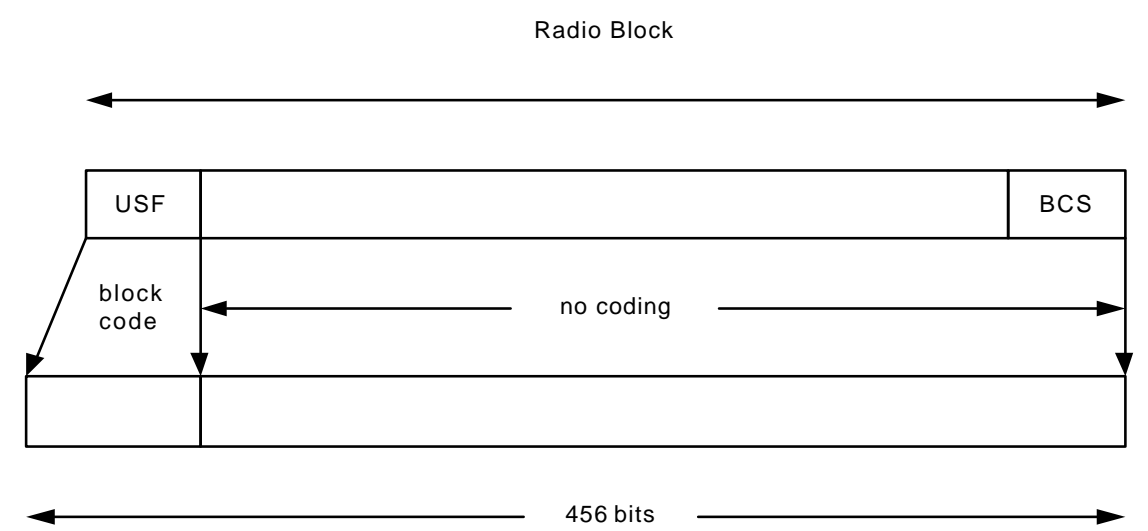

Fig. 3.- Structure of the scheme 
The next step in the simulation process is the transmission of the processed data through the wireless channel. The channel model used is based on a Ricean model [10].

The second part of the implemented GPRS simulation deals with the receiver aspects. At the reception end, the received signal is processed by the GMSK demodulator. A de-burst formatting and de-mapping block are then implemented for extracting the additional bits inserted in the transmission process. After theses blocks, the received packet is de-interleaved, rate matched, and decoded by Viterbi hard-decision decoder [3] respectively. The next process is the extraction of tail, $\mathrm{BCS}$, and USF bits. The resulting data sequence is compared with the original transmitted data sequence to check for errors.

\section{CHANNEL}

Radio waves propagate through space as traveling electromagnetic (EM) waves. The energy of signals exists in the form of electrical (E) and magnetic $(\mathrm{H})$ fields. Both electrical and magnetic fields vary with time. The two fields always exist together because a change in electrical field generates a magnetic field and a change in magnetic field develops an electrical field. Thus there is a continuous flow of energy from one field to the other.

Radio waves arrive at a mobile receiver from different directions with different time delays. They combine via vector addition at the receiver antenna to give a resultant signal with large or small amplitude depending upon whether the incoming waves combine to strengthen each other or cancel each other. As a result, a receiver at one location may experience a signal strength tens of $\mathrm{dB}$ different from a similar receiver located only a short distance away. As a mobile moves from one location to another, the phase relationships between the various incoming waves also change. Thus, there are substantial amplitude and phase fluctuations, and the signal is subjected to fading. It should also be noted that, whenever relative motion exists, there is a Doppler shift in the received signal. In the mobile radio case, the fading and Doppler shift occur as a result of the motion of the receiver through a spatially varying field. Doppler shift dso results from the motion of the scatterers of the radio waves (e.g., cars, trucks, vegetation). The effect of multipath propagation is to produce a received signal with amplitude that varies quite substantially with location. At UHF and higher frequencies, the motion of the scatterers also causes fading to occur even if the mobile set or handset is not in motion [2].
In the microcell systems, the received signal is composed of a strong direct wave and many reflected, scattered, or diffracted waves because line of sight (LOS) conditions are dominant in such systems. When the transmitted signal is given by

$$
s_{T}(t)=\operatorname{Re}\left[m(t) \exp \left(j 2 \pi f_{c} t\right)\right]
$$

a direct wave with amplitude $A_{d i r}$ is arriving at angle of $\theta_{d i r}$, and other components are expressed as the complex random Gaussian variables, the received signal can be expressed as

$$
\begin{aligned}
& s_{R}(t)=\operatorname{Re}\left[m(t) \exp \left(j 2 \pi f_{c} t\right)\right] \\
& +\operatorname{Re}\left[\sum_{i=\infty}^{\infty} A_{i} m(t) \exp \left\{j 2 \pi\left(f_{c}+f_{d} \cos \theta_{i}\right) t+\phi_{i}\right\}\right] \\
& =\operatorname{Re}\left[\left\{A_{\text {dir }} \exp \left(j 2 \pi f_{d} \cos \theta_{\text {dir }} t\right)+c_{\text {rand }}(t)\right\} m(t) \exp \left(2 \pi f_{c} t\right)\right] \\
& =\operatorname{Re}\left[c(t) m(t) \exp \left(j 2 \pi f_{c} t\right)\right]
\end{aligned}
$$

where $c_{\text {rand }}(t)$ is the variation of the nondirect waves expressed as

$$
\begin{gathered}
c_{\text {rand }}(t)=c_{\text {randI }}+j c_{\text {rand } Q}(t) \\
c_{\text {randI }}(t)=\sum_{i=-\infty}^{\infty} A_{i} \cos \left(2 \pi f_{d} \cos \theta_{i} t+\phi_{i}\right) \\
c_{\text {rand } Q}(t)=\sum_{i=-\infty}^{\infty} A_{i} \sin \left(2 \pi f_{d} \cos \theta_{i} t+\phi_{i}\right)
\end{gathered}
$$

and fading variation $c(t)$ is given by

$$
c(t)=A_{d i r} \exp \left(j 2 \pi f_{d} \cos \Theta_{d i r} t\right)+c_{\text {rand }}(t)
$$

In the case of Ricean fading, the power spectrum density of $c(t)$ is given by

$$
S(f)=b_{d i r} \boldsymbol{\delta}\left(f_{d} \cos \boldsymbol{\theta}_{d i r}\right)+\frac{b_{\text {rand }}}{\pi f_{d} \sqrt{1-\left(\frac{f}{f_{d}}\right)^{2}}}
$$


The first term in equation (7) represents a direct wave component, and the second one represents nondirect wave components. $b_{d i r}$ and $b_{\text {rand }}$ represent the power of the direct wave and the total power of the nondirect waves, and the power ration of them respectively,

$K=\frac{b_{\text {dir }}}{b_{\text {rand }}}=\frac{A_{d i r}^{2}}{2 b_{\text {rand }}}$

is called Ricean factor. Because nondirect waves are subject to a complex Gaussian random process, the probability density function of $c(t)$ is given by

$$
p\left(c_{I}, c_{Q}\right)=\frac{1}{2 \pi b_{0}^{2}} \exp \left(\frac{\left(c_{I}-A_{d i r}\right)^{2}+c_{Q}^{2}}{2 b_{0}^{2}}\right)
$$

\section{SIMULATIONSETUP}

This section describes the simulation methodology used to assess GPRS performance given in terms of Bit Error Rate (BER). During the simulation, data are transmitted continuously in a frame-by-frame (only one slot) basis over the Ricean channel. For the results shown here the channels are generated as if an MS is moving within the cell with velocities of $5 \mathrm{~km} / \mathrm{h}, 50 \mathrm{~km} / \mathrm{h}$, and $120 \mathrm{~km} / \mathrm{h}$. The simulation employs a carrier frequency of 1800 MHz.

The channel environment is changed through Ricean factor (K) since an additive white Gaussian noise (higher $\mathrm{K}$ ) until flat fading (lower $\mathrm{K}$ ). This variation is made in $\mathrm{dB}$, employing a step of $1 \mathrm{~dB}$.

The selection of data rates to be simulated is chosen to match the data rates of the GPRS data coding scheme of $9.05 \mathrm{kbps}, 13.4 \mathrm{kbps}, 15.6 \mathrm{kbps}$, and $21.4 \mathrm{kbps}$. No antenna diversity technique is considered for the simulation.

\section{SIMULATIONRESULTS}

This section describes the simulation results obtained for the simulation setups. Fig. 4 to Fig. 6 shows some results for BER as a function of $\mathrm{K}$ (Ricean factor) for user's typical mobility environment.

Fig. 4 shows the BER performance for low mobility CS1-CS4 data rate. We note that, as expected, the higher the direct wave (Ricean factor) the better the performance. Besides, CS1-CS4 presents the same results and this is due to increased influence of the long fading periods experimented by the mobile in low speed.

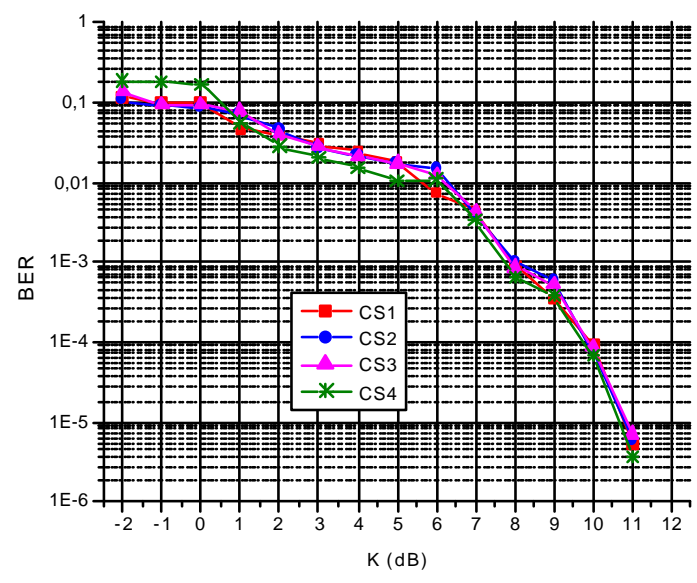

Fig. 4.- BER for user's low mobility $(\mathrm{v}=5 \mathrm{Km} / \mathrm{h})$

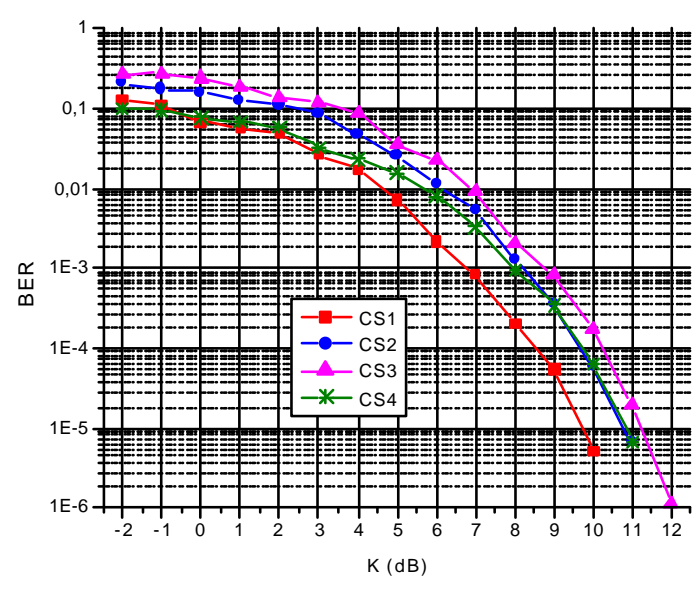

Fig. 5.- BER for user's medium mobility $(\mathrm{v}=50 \mathrm{Km} / \mathrm{h})$

Through Fig. 5, we observe that the CS1 coding scheme performs better than CS2-CS4. In this scenario, the observed performance difference is due to the higher coding gains obtained with the use the convolutional code and no puncturing in the transmission. In spite of no convolutional code in CS4, it shows a better performance than CS2-CS3 coding scheme. Analogically this is due to no puncturing process in the CS4 coding scheme.

Fig. 6 shows the BER performance for high mobility CS data rate. Note the improvement for CS1-CS3 scheme coding taking into account the medium mobility environment. In high mobility scenarios the convolutional code compensates the puncturing process 
for CS2 and CS3 scheme coding. As the CS4 does not perform the convolutional code, its performance remains the same.

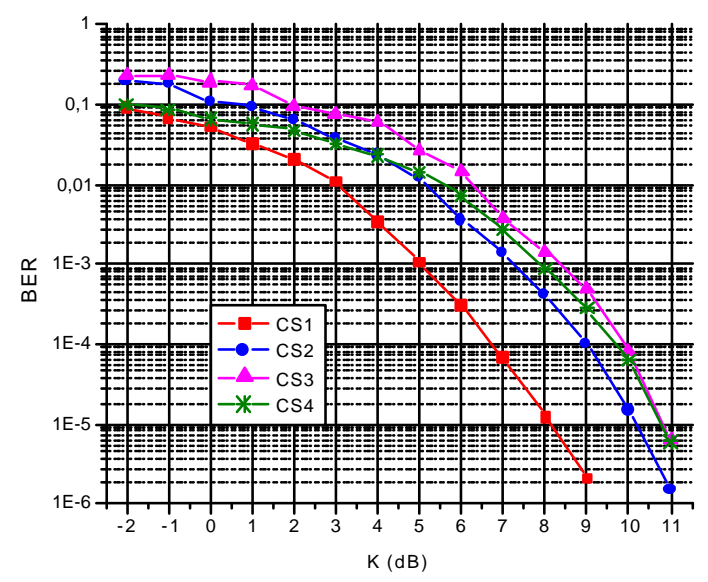

Fig. 6.- BER for user's high mobility $(v=120 \mathrm{Km} / \mathrm{h})$

\section{CONCLUSION}

The proposed and described implementation of the GPRS uplink link has been carried out successfully. For the implementation, the simulation was implemented within Mathworks/Simulink using C language to optimize the simulation performance. The simulator $\mathrm{C}$ code was aggregated to Simulink using SFunctions compiled as dlls (Dynamic Link Libraries) called from with Simulink interface. The Simulink framework environment provides a quick and simple interface that can be used for a reliable insight into the GRPS uplink behavior.

The observed differences in the BER performance according to the MS velocity are mainly dependent upon the radio propagation environment conditions.

We have shown that the simulator can be efficiently employed as a test bed for new technologies based on the GSM specification standards.

Taking into account the time spent in the simulation, the lower the BER the longest was the simulation time. For BER in the range of $10^{-6}$ ( Pentium 4 processor and 512 MB RAM) the time elapsed was 30 hours.

\section{REFERENCES}

[1] G. Brasche and B. Walker, "Concepts, Service, and Protocols of new GSM Phase 2+ General Radio
Service", IEEE Communications Magazine, pp. 94-104, August 1997.

[2] R. H. Clarke, "Statistical Theory of Mobile Radio Reception", Bell System Technical Journal 47, July-August 1968, pp.957-1000.

[3] T. Irnich and P. Stuckmann, "Analytical performance evaluation of Internet access over GPRS and its comparison with simulation results", Personal, Indoor and Mobile Radio Communications, vol. 5 , pp. 15-18, Sept. 2002.

[4] W. Featherstone and D. Molkdar, "Capacity benefits of GPRS coding schemes CS-3 and CS-4", 3G Mobile Communication Technologies, pp.287-291, May, 2002.

[5] G. Divsalar, M. K Simon, and J. H. Yuen, "Trellis Coding with Asymmetric Modulation", IEEE Transaction Communication, vol. Com 35, no.2, February 1987.

[6] GSM Specification Series 05.01, V3.3.2, "Physical Layer on the Radio Path: General Description", 1991,www.3gpp.org/ftp/Specs

[7] GSM Specification Series 05.02, V3.8.0, "Multiplexing and Multiple Access on the Radio Path", 1991, www.3gpp.org/ftp/Specs

[8] GSM Specification Series 05.03, V3.6. 1, "Channel Coding", 1991, www.3gpp.org/ftp/Specs

[9] GSM Specification Series 05.04, V3.1.2, "Modulation", 1991, www.3gpp.org/ftp/Specs

[10] Hata, M., "Empirical Formula for Propagation Loss in Land Mobile Radio Services", IEEE Transactions on Vehicular Technology 29(3), 1980. 